\title{
ROLE OF PREGABALIN IN POSTOPERATIVE PAIN MANAGEMENT IN PATIENTS UNDERGOING TOTAL KNEE ARTHROPLASTY
}

\section{Orthopaedics}

\author{
Ms. Shibila Shirin \\ K.C
}

\section{M. pharm, Pharmacy practice, Amrita school of pharmacy, Kochi}

\author{
Dr. Balu C Babu*
}

MS, DNB, Assistant Professor, Orthopaedics, Amrita Institute of Medical Sciences, Kochi *Corresponding Author

\begin{tabular}{ll}
\hline Ms. Anila K N & Assistant Professor, Amrita school of pharmacy, Kochi \\
\hline Ms. Jayasree P M & Microbiology, Amrita Institute of Medical Sciences, Kochi \\
\hline $\begin{array}{l}\text { Dr. Premila Hari } \\
\text { Mohan }\end{array}$ & Child development(PMR), Amrita Institute of Medical Sciences, Kochi
\end{tabular}

\begin{abstract}
Acute pain is the most common complication after total knee arthroplasty(TKA) causing delayed mobilization and increased demands of morphine, leading to higher operative cost. Generally opioids and NSAIDs are used but the adverse effects limit their use. So a non-opioid agent like pregabalin is often recommended. Pregabalin can significantly improve pain scores, physical functions, satisfaction and reducing chronic neuropathic pain and the requirement of other analgesics in patient undergoing TKA. Patients received either standard analgesics as per the existing hospital policy (group A) or pregabalin added to the standard analgesics(group B). Pregabalin $(75 \mathrm{mg}$ ) was given as one capsule, the last night before the surgery and continued one capsule twice a day until end of postoperative day (POD) 14 and one capsule at bedtime POD15, POD16. The group B showed significant reduction in pain score after 2 weeks of the surgery. It was $3.20 \pm 1.03$ in group A and $2.48 \pm 0.77$ in group B. The mean comparison of satisfaction level in group A and group B was found to be $7.80 \pm 0.96$ and $9.52 \pm 1.04$ respectively. The mean comparison of incidence of neuropathic pain in group $A$ and group B was found to be $6.70 \pm 4.01$ and $4.24 \pm 4.14$ respectively. Our study concluded that pregabalin is effective in reducing pain after 2 weeks post TKA, and incidence of neuropathic pain. Increased patient satisfaction level and the quality of life of patients were observed in pregabalin treated group.
\end{abstract}

\section{KEYWORDS}

Arthroplasty, Knee, Analgesia, Pregabalin.

\section{INTRODUCTION}

Osteoarthritis (OA) is the most common degenerative joint disease and a significant cause of pain and disability in adults 1,2 . OA is the wearing down of articular cartilage that affects the entire diarthrodial joint, which includes articular cartilage, synovium and subchondral bone with adjacent muscles and ligaments. Any degeneration to the structure or any alterations to the functioning of these articulating tissues produces clinical OA3. Pain is the major and most devastating part of OA. In the initial stages of OA, pain is aggravated during joint motion, and it may be relieved by rest. However, in the advanced stage, there may be rest pain 4 . Acute or chronic insults such as age, obesity, normal wear and tear, traumatic joint injuries may initiate the imbalance between matrix synthesis and matrix degradation. These changes may initiate the subchondral bone to undergo pathological changes and thereby activate the vasopeptides and matrix metalloproteinase (MMP) 5. In the initial stage, cartilage slowly becomes callous and thin, and the bone beneath the cartilage becomes thick. Later, there will be an abnormal stimulation of these biochemical and metabolic factors altering the tissue homeostasis of articular cartilage by neovascularisation and increasing the permeability of adjacent cartilage. This may accelerate the structural changes like cartilage loss, and joint space narrowing thus leading to painful and deformed joint6.

Since there is no complete cure for OA, the main goal of the therapy is to alleviate the signs and symptoms and slow down the progression of the disease. The treatment of OA varies from physiotherapy, orthopaedic aids and orthoses, pharmacotherapy and finally the surgery and rehabilitation 7 .

Total knee arthroplasty (TKA) is a surgical procedure whereby the diseased knee joint surface is replaced with artificial material. TKA is very effective in restoring joint function, improving the pain and health-related quality of life $(\mathrm{QoL})$ of patients8. TKR is always associated with severe to moderate pain after the surgery9. Pang et al. reported that the severity of pain in TKA is more than other orthopaedic surgeries 10. Clinical studies indicate that severe postoperative pain is associated with an increased risk of complications, slowing the rehabilitation process, delays the improvement of persistent pain states, prolongs the length of hospital stay, and raises the overall treatment costs. Therefore, appropriate pain management protocol is necessary to relieve postoperative pain and achieve early functional recovery11. Opioids and NSAIDs have generally been used for pain control after TKA. However, these medications may cause serious side effects, especially in geriatric patients. Opioid-related complications are sedation, dizziness, nausea, vomiting, constipation, physical dependence, tolerance, respiratory depression. Anaemia, stomach ulcers, dizziness, swelling in the legs, abnormal liver tests (blood tests), headaches, easy bruising, ringing in the ears, rash are serious problems with NSAIDs. Hence, these adverse effects limit the use of opioids and NSAIDs 12

Recent studies reported that $\alpha 2 \mathrm{~d}$ subunit calcium channel ligands, like gabapentin and pregabalin, may be useful in providing effective postsurgical analgesia. They diminish the central sensitisation by calming down hyperexcited dorsal horn neurons. This discovery introduces the possibility of using such drugs in the peri-operative setting to counter the neuropathic component of post-surgical pain. Although pregabalin was approved for the treatment of partial seizures, it has also been used for other indications, such as fibromyalgia, diabetic neuropathy, and even acute post-surgical pain 13 .

Pregabalin is a more potent analogue of gabapentin with better oral bioavailability and potentially more consistent clinical effects. It can be used for postoperative pain management because it is welltolerated, does not cause any gastrointestinal bleeding and also reduces the morphine related complications 14,15 .

The primary objective of our study is to assess the role of pregabalin as an adjuvant in pain management and evaluate the clinical outcomes associated with it in patients undergoing total knee arthroplasty. The secondary objectives are to assess the clinical outcomes and the incidence of neuropathic pain in TKA patients after three months postsurgery. Also to evaluate the level of patient satisfaction on the use of pregabalin as an adjuvant in pain management and its effects in diabetic patients undergoing TKA.

\section{MATERIALAND METHODS}

We did a prospective observational study of patients undergoing TKA in our tertiary care centre during the study period September 2018 to 
April 2019 to after obtaining ethical clearance and informed consent. We included patients aged above 40 years who underwent primary TKA and excluded patients with allergy or intolerance to the study medication(pregabalin), hepatic failure/ chronic renal failure, uncontrolled diabetes mellitus (HbAlc >8) and who used gabapentin/pregabalin regularly for longer than three months.

Patients were divided into two groups A and B by the surgeon randomly. Group A patients received standard institutional analgesic protocol for TKA, and Group B patients received pregabalin in addition to the standard protocol. The standard analgesic protocol included Inj. Para (Paracetamol 1g), Inj. Ropivacaine $2 \%$ via adductor canal block, Inj. Fentanyl, Rupatch(Buprenorphine 10mg transdermal patches) in the immediate postoperative period and first postoperative day (POD). T. Etrobax (Etoricoxib 60mg) and T. Ultracet (Tramadol + Acetaminiphen 2 tab) were added on POD 2 after the adductor block was removed.For group B patients pregabalin was given as one capsule $(75 \mathrm{mg})$, the night before the surgery and continued giving one capsule twice a day until the end of the postoperative day (POD) 14 and one capsule at bedtime POD15, POD16.

Preoperative data, including the patient demographics: age, sex, body mass index(BMI), pain scores and the Knee Injury and Osteoarthritis Outcome Score (KOOS) were noted during direct patient interview by a blinded observer. Subsequent data were collected, which included the postoperative pain assessment on POD1, POD2, POD3 and at two weeks using Numeric Rating Scale (NRS). After two weeks of surgery, satisfaction with pain management was assessed. The occurrence of neuropathic pain and orthopaedic outcomes were assessed three months after surgery. Neuropathic pain was evaluated by using the Leeds Assessment of Neuropathic Symptoms and Signs (LANSS). Orthopaedic outcomes were evaluated by using KOOS.

\section{STATISTICAL METHOD}

Statistical analysis was performed using IBM SPSS version 20.0 software. Categorical variables are expressed using frequency and percentage, and continuous variables expressed using mean and standard deviation. To test the statistical significance of the comparison of categorical variables between two groups, Chi-square test was used. To test the statistical significance of the difference in the mean values of continuous variables between two groups, Student's ttest/Mann Whitney U test was used. To test the statistical significance of the difference in the mean values of preoperative and postoperative variables between two groups, Paired t-test/ Wilcox on Signed rank test was used.

\section{RESULTS}

In our study, we analysed a total of 55 patients who underwent TKA, of which 30 were in the group $\mathrm{A}$, and the remaining 25 were in the group $\mathrm{B}$. We found that in both the study groups females were predominant than males. The majority of patients were in the age of sixties. There were no statistically significant differences between the groups based on baseline characteristics ( $p$-value $>0.05$ ). (Table 1).

Table 1: Demographics and characteristics of participants

\begin{tabular}{|c|c|c|}
\hline & Group A $(\mathbf{n}=\mathbf{3 0})$ & Group B $(n=25)$ \\
\hline \multicolumn{3}{|c|}{ Age } \\
\hline \multicolumn{3}{|c|}{ Age group, n (\%) } \\
\hline $40-49$ & $1(3.3)$ & 0 \\
\hline $50-59$ & $4(13.3)$ & $7(28)$ \\
\hline $60-69$ & $15(50)$ & $10(40)$ \\
\hline $70-79$ & $10(33.3)$ & $6(24)$ \\
\hline $80-89$ & & $2(8)$ \\
\hline \multicolumn{3}{|c|}{ Gender } \\
\hline \multicolumn{3}{|c|}{ Gender, n (\%) } \\
\hline Male & $6(20)$ & $3(12)$ \\
\hline Female & $24(80)$ & $22(88)$ \\
\hline \multicolumn{3}{|c|}{ Diabetes Mellitus(DM) } \\
\hline \multicolumn{3}{|c|}{ Patients having DM, n (\%) } \\
\hline Male & $6(20)$ & $6(24)$ \\
\hline Female & $24(80)$ & $19(76)$ \\
\hline \multicolumn{3}{|c|}{ Preoperative KOOS Score } \\
\hline Pain & $78.21 \pm 7.40$ & $79.28 \pm 7.64$ \\
\hline Symptoms & $69.23 \pm 5.31$ & $67.91 \pm 8.87$ \\
\hline ADL & $65.52 \pm 6.45$ & $66.64 \pm 4.38$ \\
\hline QOL & $91.25 \pm 6.68$ & $90.75 \pm 6.01$ \\
\hline Global score & $80.40 \pm 4.05$ & $80.55 \pm 3.24$ \\
\hline
\end{tabular}

ASSESSMENT OF PAIN IN STUDY POPULATION

Although the mean pain score before surgery, POD 1, POD 2 and POD 3 was similar in both groups, the group B, i.e. with pregabalin showed a significant reduction in pain score after two weeks of the surgery. It was $3.20 \pm 1.03$ in group $A$ and $2.48 \pm 0.77$ in group B with a p-value of 0.006 .

Figure 1: Mean comparison of pain between groups Mean comparison of pain scores between groups

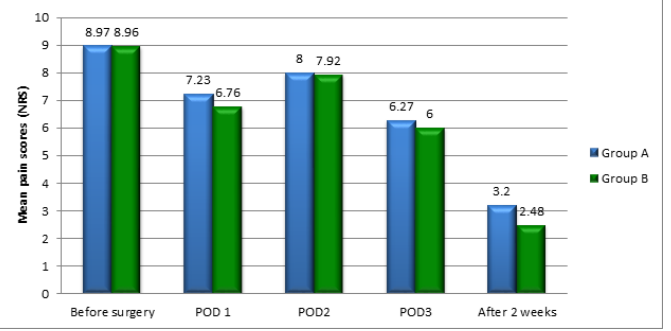

Assessment Of Satisfaction In Study Populations

The satisfaction level of patients who underwent the TKA was assessed by using a self-designed standardised satisfaction scale. The mean comparison of satisfaction in group A and group B was found to be $7.80 \pm 0.96$ and $9.52 \pm 1.04$, respectively. That is the pregabalin treated groups showed much higher satisfaction level than the other group.

Figure 2: Mean comparison of satisfaction between groups

Mean comparison of satisfaction between groups

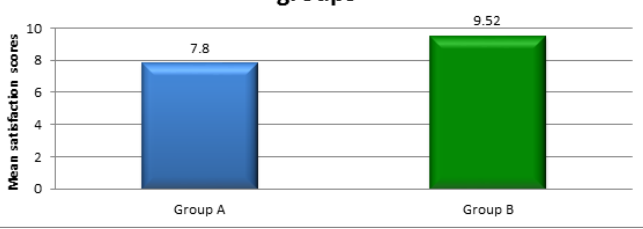

In group A, 2(16.6\%) patients were very satisfied, $23(76.66 \%)$ patients were satisfied, and $5(16.66 \%)$ patients were slightly satisfied. However, in group B, those who treated with pregabalin along with standard analgesics, $20(80 \%)$ patients' shows very satisfied, $4(16 \%)$ patients were satisfied, and $1(4 \%)$ patient was slightly satisfied.

Table 2: Comparison of satisfaction level of pain relief after surgery in study population

\begin{tabular}{|c|c|c|}
\hline Satisfaction level & $\begin{array}{c}\text { Usual analgesics, } \\
\mathbf{n = 3 0}(\mathbf{\%})\end{array}$ & $\begin{array}{c}\text { Analgesic and pregabalin } \\
\text { group, } \mathbf{n = 2 5}(\mathbf{\%})\end{array}$ \\
\hline Slightly satisfied & $5(16.66)$ & $1(4)$ \\
\hline Satisfied & $23(76.66)$ & $4(16)$ \\
\hline Very satisfied & $2(6.66)$ & $20(80)$ \\
\hline
\end{tabular}

Assessment Of Incidence Of Neuropathic Pain In Study Population

The incidence of neuropathic pain in the study population was evaluated using the LANSS scale. The mean comparison of the incidence of neuropathic pain group A and group B was found to be $6.70 \pm 4.01$ and $4.24 \pm 4.14$, respectively. That is group A without pregabalin shows much more incidence of neuropathic pain than the other group.

Figure 3: Mean comparison of incidence of neuropathic pain between groups

\section{Mean Comparison of LANSS Score} between groups

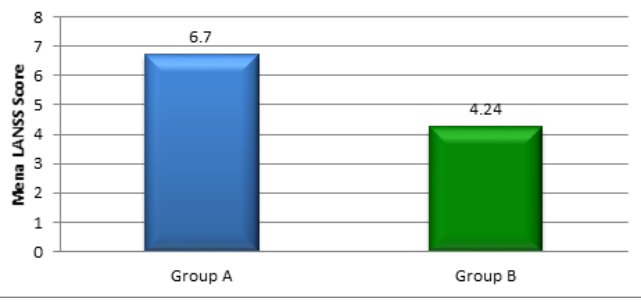

International Journal of Scientific Research 


\section{Assessment Of Incidence Of Allodynia In Study Population}

The incidence of allodynia in the operated leg was lower in group B with pregabalin. In group A, allodynia was found in $4(13.3 \%)$ patients. But in group B, those who treated with pregabalin along with usual analgesics, only $2(8 \%)$ patients show allodynia.

Table 3: Assessment of incidence of allodynia in study population

\begin{tabular}{|c|c|c|}
\hline Allodynia & $\begin{array}{c}\text { Usual analgesics, } \\
\mathbf{n}=\mathbf{3 0}(\mathbf{\%})\end{array}$ & $\begin{array}{c}\text { Analgesic and pregabalin } \\
\text { group, } \mathbf{n}=\mathbf{2 5}(\mathbf{\%})\end{array}$ \\
\cline { 2 - 3 } & $4(13.3)$ & $2(8)$ \\
\hline
\end{tabular}

Assessment Of Quality Of Life In Study Populations

The assessment of QoL in the study population was evaluated by using the KOOS scale. The mean global score of QoL in group A and group B at baseline was found to be $80.40 \pm 4.05$ and $80.55 \pm 3.24$ respectively and the after three months it was found to be $46.77 \pm 2.66$ and $44.55 \pm 4.35$ respectively with a p-value of 0.031 . Also, in pregabalin treated group, there was a significant improvement in the pain of patients after three months of surgery. The mean pain score in group A and group B after three months was found to be $36.38 \pm 4.57$ and $34.11 \pm 4.20$, respectively, with a p-value of 0.061 . So the patients who were administered with the pregabalin as an adjuvant along with usual analgesics showed more improvement in knee function than the other group. The mean of pain and global scores in the KOOS scale shows statistically significant difference and hence, showed improvement in QoL. The two groups also showed a significant improvement in QoL of patients from baseline to 3 months after the surgery.

Figure 4: Mean comparison of KOOS score between groups after 3 months

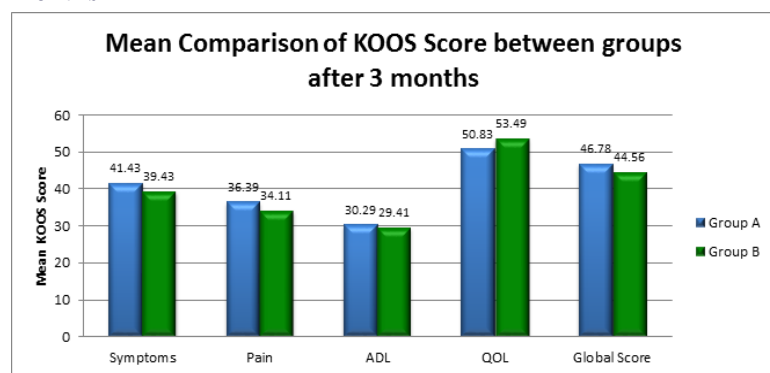

Mean Comparison Of Pre And Post Koos Score Within Drug Group

There was a significant improvement in quality of life of patients from baseline to 3 months after the surgery with a $p$ value of 0.000 in each group.

Table 4: Mean comparison of pre and post KOOS score within drug group

\begin{tabular}{|c|c|c|c|c|}
\hline Drugs & $\begin{array}{c}\text { KOOS } \\
\text { domains }\end{array}$ & Timeline & Mean \pm SD & $\begin{array}{c}p \\
\text { value }\end{array}$ \\
\hline \multirow{10}{*}{$\begin{array}{l}\text { Group A - } \\
\text { Standard } \\
\text { analgesics }\end{array}$} & \multirow[t]{2}{*}{ Symptoms } & At baseline & $78.21 \pm 7.40$ & \multirow[t]{2}{*}{0.000} \\
\hline & & After 3 months & $41.42 \pm 4.74$ & \\
\hline & \multirow[t]{2}{*}{ Pain } & At baseline & $69.23 \pm 5.31$ & \multirow[t]{2}{*}{0.000} \\
\hline & & After 3 months & $36.38 \pm 4.57$ & \\
\hline & \multirow[t]{2}{*}{$\mathrm{ADL}$} & At baseline & $65.52 \pm 6.45$ & \multirow[t]{2}{*}{0.000} \\
\hline & & After 3 months & $30.21 \pm 3.24$ & \\
\hline & \multirow[t]{2}{*}{ QOL } & At baseline & $91.2 \pm 6.68$ & \multirow[t]{2}{*}{0.000} \\
\hline & & After 3 months & $50.8 \pm 6.30$ & \\
\hline & \multirow[t]{2}{*}{ Global score } & At baseline & $80.40 \pm 4.05$ & \multirow[t]{2}{*}{0.000} \\
\hline & & After 3 months & $46.77 \pm 2.66$ & \\
\hline \multirow{10}{*}{$\begin{array}{l}\text { Group B- } \\
\text { Standard } \\
\text { analgesics with } \\
\text { Pregabalin }\end{array}$} & \multirow[t]{2}{*}{ Symptoms } & At baseline & $79.28 \pm 7.64$ & \multirow[t]{2}{*}{0.000} \\
\hline & & After 3 months & $39.42 \pm 8.40$ & \\
\hline & \multirow[t]{2}{*}{ Pain } & At baseline & $67.91 \pm 8.87$ & \multirow[t]{2}{*}{0.000} \\
\hline & & After 3 months & $34.11 \pm 4.20$ & \\
\hline & \multirow[t]{2}{*}{$\mathrm{ADL}$} & At baseline & $66.64 \pm 4.38$ & \multirow[t]{2}{*}{0.000} \\
\hline & & After 3 months & $29.41 \pm 4.98$ & \\
\hline & \multirow[t]{2}{*}{ QOL } & At baseline & $90.7 \pm 6.01$ & \multirow[t]{2}{*}{0.000} \\
\hline & & After 3 months & $53.49 \pm 5.12$ & \\
\hline & \multirow[t]{2}{*}{ Global score } & At baseline & $80.55 \pm 3.24$ & \multirow[t]{2}{*}{0.000} \\
\hline & & After 3 months & $44.55 \pm 4.35$ & \\
\hline
\end{tabular}

Comparison Of Pain Score Diabetes Mellitus Within Drug Groups A total of 10 patients only had DM, 4 were from group A and 6 from the group B. There was no statistical significance between these two groups till POD 3. However, after two weeks, those patients who took the pregabalin along with analgesics shows much less pain score than the other group without pregabalin.

\section{Figure 5: Comparison of pain score in patients having DM}

\section{Comparison of pain scores in diabetic patients}

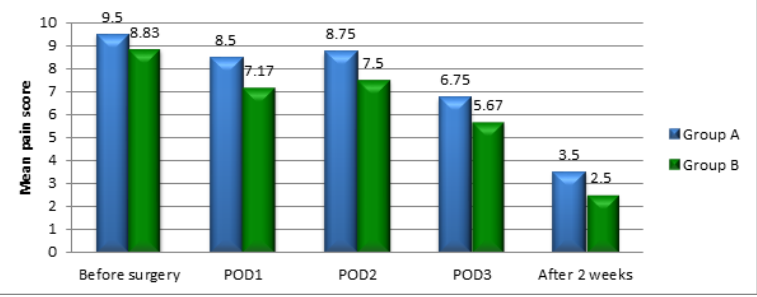

A total of 45 patients without DM, 26 were from group A and 19 from the group B. There was a statistically significant difference between these two groups after two weeks. The patients who took the pregabalin along with analgesics shows much less pain score than the other groups who did not take the pregabalin.

Figure 6: Comparison of pain score in patients without diabetic mellitus

\section{Comparison of pain scores in nondiabetic} patients

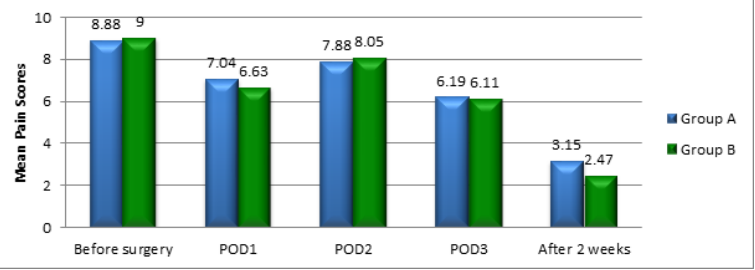

Mean Comparison Of Lanss Score With Respect To Diabetes Mellitus Within Drug Groups

There was a statistically significant difference between the two groups in the incidence of neuropathic pain. Those patients who took the pregabalin along with analgesics shows much less LANSS score than the other group who did not take pregabalin.

A total of 45 patients without DM, 26 were from group A, and 19 from the group B. LANSS score between the two groups of the patient without DM showed a significant difference in the incidence of neuropathic pain. Those patients who took the pregabalin along with analgesics shows a lower incidence of neuropathic pain than the other group who did not take the pregabalin.

Table 5: Mean comparison of LANSS score with respect to diabetes mellitus within drug groups

\begin{tabular}{|c|c|c|c|}
\hline $\begin{array}{c}\text { Patient } \\
\text { having DM }\end{array}$ & $\begin{array}{c}\text { Usual } \\
\text { Analgesics, } \\
\text { n }(\mathbf{M e a n} \pm \text { SD) }\end{array}$ & $\begin{array}{c}\text { Analgesics and } \\
\text { Pregabalin, } \\
\text { n (Mean } \pm \text { SD) }\end{array}$ & p-value \\
\hline Yes & $4(7.25 \pm 3.50)$ & $6(5.00 \pm 5.67)$ & $0.032^{*}$ \\
\hline No & $26(6.62 \pm 4.13)$ & $19(4.0 \pm 3.71)$ & $0.034^{*}$ \\
\hline
\end{tabular}

\section{DISCUSSION}

Total knee arthroplasty (TKA) is one of the most common surgical procedures in the orthopaedic field. Effective postoperative pain management is necessary to promote recovery and to prevent complications 16 . In recent years, some randomised controlled trials (RCTs) have been carried out to evaluate the effects of pregabalin in postoperative pain management. However, different conclusions have been derived on the efficacy of pregabalin for pain management among TKA patients. Some studies 17,18 reported that perioperative administration of pregabalin reduced opioid consumption improved postoperative analgesia, and yielded higher patient satisfaction levels. However, certain other studies 19, 20, 21 showed that there was no significant difference between pregabalin and placebo with respect to the pain after TKA. In regards to this existing controversial outcome of pregabalin use in TKA, our study explored the actual scenario in our study settings. 
In our study on TKA patients, we found that the mean pain score before surgery, POD 1, POD 2 and POD 3 was similar in both groups. The pregabalin group showed a significant reduction in pain score after two weeks of the surgery. The results were further supported by the studies conducted by J. T. YaDeau et al. 20 and Jing Hui Yik et al22. The pregabalin group showed a significant reduction in pain score after two weeks of the surgery. This result was consistent with another study conducted by H. Clarke et al19 in THA (Total Hip Arthroplasty) patients. Their study stated that pregabalin reduced the daily pain scores and adjunct opioid consumption for one week after discharge.

Our study also assessed the satisfaction level of patients who underwent the TKA. The mean comparison of satisfaction level in pregabalin treated group was found to be higher. The result was consistent with the studies conducted by Pradeep Jain et al17 and contrary to the study conducted by J. T. YaDeau et al20, in which they stated that pregabalin decreases patient satisfaction level.

The incidence of neuropathic pain in the study population was evaluated by using the LANSS scale. The mean comparison of the incidence of neuropathic pain was less with pregabalin treated group. A similar conclusion was reported by Buvanendran A et al23. They conducted a trial to examine whether pregabalin given before and for 14 days after TKA will reduce the incidence of neuropathic pain. We also assessed the incidence of allodynia in the operated leg, which was less with patients on pregabalin $8 \%$ vs $13.4 \%$ in patients without pregabalin.

The assessment of QoL in the study population was evaluated by using the KOOS scale. KOOS Scale is an instrument to assess the patient's condition about the knee and associated problems. A normalised score (100 indicating no symptoms and 0 indicating extreme symptoms) is calculated for each subscale. A lower score on KOOS indicates worsening of symptoms, pain, activities of daily living, function in sport and QoL. But, here we present the KOOS score differently than normal. 0 represents an ideal situation, while 100 is the worst situation. Also, here we intentionally omitted the questions regarding the function, sports and recreational activities since most of the patients are older age (above 60) and were not willing to answer those questions. The mean global score of QoL in group A and group B was better at three months after surgery with a p-value of 0.031 . Also, in pregabalin treated group, significant improvement in pain was observed after three months after surgery. So the patients who were administered with pregabalin as an adjuvant along with usual analgesics showed more improvement in knee function than the other group. The mean of pain and global scores in the KOOS scale shows statistically significant difference and hence, showed improvement in QoL.The study was further supported by the studies conducted by Lara G et al. 24, Kadic L et al. 25 and Asokumar Buvanendran et al. 23

We also evaluated the effect of pregabalin in DM patients who underwent TKA. By analysing the patients with and without diabetes, a statistically significant difference was observed in pain scores and LANSS scores in the group treated with pregabalin.

The major limitation of the present study was that the actual effectiveness of pregabalin could not be established due to the additive effect of other analgesics. Further studies should focus on the studies regarding the effectiveness of different doses of pregabalin in postoperative pain management, and the effectiveness of pregabalin on postoperative pain in subgroup population like diabetes, sleep apnoea with larger sample size can be designed.

\section{CONCLUSION}

Our study concluded that pregabalin is effective in reducing postoperative pain at two weeks after TKA, and also decreased the incidence of neuropathic pain. Increased patient satisfaction level and the quality of life of patients were observed in the pregabalin treated group. In analysing the role of pregabalin in TKA with diabetic patients showed improved pain scores and decreased incidence of neuropathic pain as compared with non-diabetic TKA patients.

\section{REFERENCES}

1. Zhang Y, Jordan JM. Epidemiology of osteoarthiritis. Clin Geriatr Med. 2010; 26(3): Zhang Y,

. Murphy L, Helmick CG. The impact of osteoarthritis in the United States: A populationhealth perspective. Am J Nurs. 2012: 112 (3 Suppl 1): S13-S19.

3. Dagenais S, Garbedian S, Wai EK. Systematic review of the prevalence of radiographic primary hip osteoarthritis. Clin Orthop Relat Res. 2009; 467(3): 623-637

4. Hawker GA, Stewart L, French MR, et al. Understanding the pain experience in hip an knee osteoarthritis-an OARSI/OMERACT initiative. Osteoarthritis Cartilage 2008; 16(4):415-22.

5. Mandelbaum B, Waddell D. Etiology and Pathophysiology of Osteoarthritis Orthopedics. 2005; 28(2): S207-S214.

6. Iannone F, Lapadula G. The pathophysiology of osteoarthritis. Aging Clin \& Exp Res 2003; 15(5):364-372

7. Mohing W. Die Arthrosis Deformans des Kniegelenks. Berlin: Springer-Verlag; 1966

8. Fischer HB, Simanski CJ, Sharp C et al (2008) A procedure-spe-cific systematic review and consensus recommendations for post-operative analgesia following total knee arthroplasty. Anaesthesia 63(10): 1105-1123

9. A. Osawa, K. Yoshida, H. Kanazawa, Y. Maruyama, K. Kaneko. Efficacy of periarticula A. Osawa, K. Yoshida, H. Kanazawa, Y. Maruyama, K. Kaneko. Efficacy of periarticular multimodal drug injection in total knee arthroplasty for pain

10. W.-W. Pang, T.-C. Hsu, C.-C. Tung, C.-P. Hung, D.-P. Chang, M.-H. Huang. Is total knee replacement more painful than total hip replacement? Acta Anaesthesiologica Sinica. 38 (3) (2000) $143-148$

11. Tayyem M, Elhadid F, Haddad R,et al. Perioperative use of pregabalin in total knee arthroplasty: meta-analysis of randomized controlled trials. J Musc Res. 2017; 20 ( 2):1750009 (8 page)

12. Horlocker TT (2010) Pain management in total joint arthroplasty: a historical review. Orthopedics 33(9 Suppl):14-19.

13. Stahl SM. Anticonvulsants and the relief of chronic pain:Pregabalin and gabapentin as alpha(2)delta ligands at voltage-gated calcium channels. J Clin Psychiatry 2004; 6:596-7.

14. Bockbrader NH, Wesche D, Miller R, et al. A Comparison of the Pharmacokinetics and Pharmacodynamics of Pregabalin and Gabapentin. Clin Pharmacokinetics. 2010;49(10): 661-669

15. Eisenberg E, River Y, Shifrin A, et al. Antiepileptic drugs in the treatment of neuropathic pain. Drugs. 2007; 67(9): 1265-89

16. Turan A, Kaya G, Karamanlioglu B, Pamukçu Z, Apfel CC. Effect of oral gabapentin on postoperative epidural analgesia. Br J Anaesth 2006; 96: 242-6.

17. Jain P, Jolly A, Bholla V, Adatia S, Sood J.Evaluation of efficacy of oral pregabalin in reducing postoperative pain in patients undergoing total knee arthroplasty.Indian J Orthop. 2012 Nov;46(6):646-52

18. Dong J, Li W, Wang Y . The effect of pregabalin on acute postoperative pain in patient undergoing total knee arthroplasty: A meta-analysis. Int J Surg. 2016 Oct; 34:148-160.

9. Han, C., Kuang, M.-J., Ma, J.-X., \& Ma, X.-L. (2017). Is pregabalin effective and safe in total knee arthroplasty? A PRISMA-compliant meta-analysis of randomized-controlled trials. Medicine (Baltimore). 2017 Jun;96(26):e6947

20. YaDeau JT, Lin Y, Mayman DJ, Goytizolo EA, Alexiades MM, Padgett DE, Kahn RL, Jules-Elysee KM, Ranawat AS, Bhagat DD, Fields KG, Goon AK, Curren J, Westrich GH. Pregabalin and pain after total knee anthroplasty: a double-blind, randomized, placebo-controlled, multidose trial. Br J Anaesth. 2015 Aug:115(2):285-93

21. Lee JK, Chung KS, Choi CH.The effect of a single dose of preemptive pregabalin administered with COX-2 inhibitor: a trial intotal knee arthroplasty.J Arthroplasty. 2015 Jan;30(1):38-42.

22. Yik, J. H., Tham, W. Y. W., Tay, K. H., Shen, L., \& Krishna, L. (2019).Perioperative pregabalin does not reduce opioid requirements in total knee arthroplasty. Knee Surg Sports Traumatol Arthrosc. 2019 Feb 9

23. Buvanendran A, Kroin JS, Della Valle CJ, Kari M, Moric M, Tuman KJ.Perioperative oral pregabalin reduces chronic pain after total knee arthroplasty: a prospective, randomized, controlled trial.Anesth Analg. 2010 Jan 1;110(1):199-207

24. Lara G, Vittorio P, Elisabetta B et al. Perioperative Pregabalin for Postoperative Pain Control and Quality of Life after Major Spinal Surgery. Journal of Neurosurgical Anesthesiology 2012; 24(2):121-126.

25. Kadic L, van Haren FG, Wilder-Smith O, Bruhn J, Driessen JJ, de Waal Malefijt MC The effect of pregabalin and s-ketamine in total knee arthroplasty patients: A randomized trial.J Anaesthesiol Clin Pharmacol. 2016 Oct-Dec;32(4):476-482 